\title{
On-the-Fly Image Quality Evaluation for Single-Particle Analysis Cryo-Electron Microscopy
}

\author{
Lingbo Yu ${ }^{1}$, Erik Franken ${ }^{1}$, Andreas Voigt ${ }^{1}$, Fanis Grollios ${ }^{1}$, Peter Tiemeijer ${ }^{1}$ and Steve Reyntjens ${ }^{1}$ \\ 1. Thermo Fisher Scientific, Materials and Structural Analysis, Eindhoven, the Netherlands
}

Structure determination of individual proteins and protein complexes by single-particle analysis cryoelectron microscopy (SPA Cryo-EM) has greatly benefited from recent advances in instrumentation and software. Most notably, the availability of stable cryo-TEM platforms, high performance direct electron detectors, the systematic automation of data collection, and the revolution in image processing software have all provided important contributions to the breakthrough of SPA cryo-EM as a generally accepted structural biology technique. Further adoption of SPA cryo-EM will depend heavily on making the best possible performance routinely accessible to more structural biologists. This requires continued improvements in robustness and ease of use. Especially the introduction of more automation and "intelligence" during the acquisition phase will be key.

Proteins and protein complexes are radiation sensitive, limiting the total dose that can be applied for each image. To overcome the low signal-to-noise ratio (SNR) due to limited dose, a large number of images of the same specimen are taken and averaged to increase the SNR. For acquisition of large amounts of data, automation is preferred to replace the repetitive steps. The biological questions of interest often require structures to be resolved at high resolution, driving the need for robust and efficient automated data collection to go on for hours, or even days.

The large amount of images that are acquired during such a session, provides opportunities for enhancing the robustness and efficiency of the data collection itself. While the analysis of individual images has limited applicability due to the low SNR of these individual images, analyzing multiple images at once provides enough information to statistically evaluate the hardware performance and robustly estimate optical parameters. This evaluation can subsequently be used to feed back into the microscope settings, optimizing the data collection. For example, in the application of the volta phase plate (VPP), the phase shift can be monitored and used as a guidance to switch to the next phase plate location [1]. At the same time, the defocuses and other optical parameters can also be measured and compared to their target values. A systematic offset, if any, can be discovered and corrected.

Here we present recent investigations into this concept, i.e. on-the-fly analysis of a number of critical optical parameters and application of the results in a closed loop system to improve the control over the acquisition process. For example, the contrast transfer function (CTF) estimation [2, 3] of an image results in two defocus value: a short axis defocus, $d f_{1}$, and a long axis defocus, $d f_{2}$. The astigmatism of an image can then be described by the difference between the two defocus values $\left(d f_{1}-d f_{2}\right) / 2$ and the angle $\theta$ i.e. the direction of $d f_{1}$. In the CTF, two main sources cause Thon rings to have an elliptical shape: the first is magnification anisotropy; and the second is objective astigmatism. Magnification anisotropy is focus-dependent; objective astigmatism is not. There are linear relations between them:

$$
\begin{aligned}
& a s t_{x}=\frac{d f_{1}-d f_{2}}{2} \cos (2 \theta)=k_{x} \frac{d f_{1}+d f_{2}}{2}+\alpha_{x}, \\
& a s t_{y}=\frac{d f_{1}-d f_{2}}{2} \sin (2 \theta)=k_{y} \frac{d f_{1}+d f_{2}}{2}+\alpha_{y},
\end{aligned}
$$


where $a s t_{x}$ and $a s t_{y}$ are two orthogonal components $x$ and $y$ of the ellipticity; $k_{x}$ and $k_{y}$ are the distortions from magnification anisotropy in $x$ - and $y$-components respectively; and $\alpha_{x}$ and $\alpha_{y}$ are the distortions from objective astigmatism in $x$ - and $y$-components respectively. The systematic objective astigmatism and the focus-dependent magnification anisotropy can be reliably measured by a linear fit, see Fig. 1. In practical situations, defocus values typically vary from $1 \mu \mathrm{m}$ to $3.5 \mu \mathrm{m}$, which is sufficient for fitting. The resulting information, estimated over a statistically relevant sample of multiple images, can be fed back to the microscope for on-the-fly optics adjustment. At the end of each automated data acquisition session, such information is also reported to the user for future analysis.

Using such on-the-fly quality evaluation scheme, the acquisition will yield higher quality raw data while requiring less user interventions.

\section{References:}

[1] R Danev, D Tegunov and W Baumeister, eLife 6 (2017).

[2] K Zhang, Journal of Structural Biology 193 (2016), p. 1-12.

[3] A Rohou and N Grigorieff, Journal of Structural Biology, 192 (2015), p. 216-221.
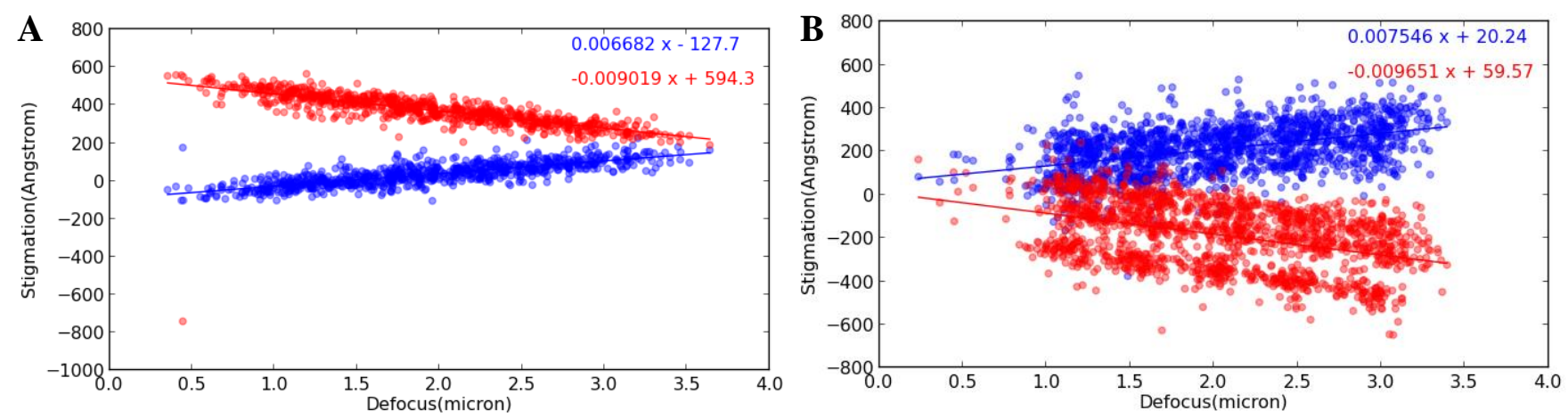

Figure 1.

(A) Dataset taken at 73000x nominal magnification with defocus range between 0.5 um to 3.5 um. Of all images the FFT was determined and CTF was fitted. Using linear fits the $\mathrm{x}$ (red) and $\mathrm{y}$ (blue) components of magnification anisotropy and astigmatism were determined (linear fit equations in inset). (B) is similar to (A), but images were taken at 3 different image/beam shift settings (multiple images per Quantifoil hole, taken at 92000x nominal magnification), resulting in 3 "clusters" of datapoints. Linear fits (only 1 shown in image) demonstrate that the magnification anisotropy is constant (slope of the linear fit), but that the objective astigmatism varies with beam/image shift (intersection at 0 defocus). This knowledge can be used to correct for objective astigmatism induced by beam/image shift. 